\title{
Efficacy and Safety of Direct-Acting Oral Anticoagulants Use in Acute Portal Vein Thrombosis Unrelated to Cirrhosis
}

\author{
Filipe Nery a, b, c, g, Diana Valadares ${ }^{\mathrm{a}, \mathrm{c}}$, Sara Morais ${ }^{\mathrm{d}}$, Manuel Teixeira Gomes ${ }^{\mathrm{c}, ~ e}$, \\ Andrea De Gottardi ${ }^{\mathrm{f}}$
}

\begin{abstract}
In acute portal vein thrombosis (APVT) unrelated to cirrhosis, anticoagulant therapy is classically started with low molecular weight heparin or vitamin $\mathrm{K}$ antagonists. New direct-acting oral anticoagulants (DOACs) are used in the treatment of venous thrombosis outside the splanchnic vascular bed, but not in the latter. We report a young female with APVT occurring in a non-cirrhotic liver linked to heterozygosity of factor V-Leiden and prothrombin G20210A gene mutations. Rivaroxaban was started, with total recanalization of the left and partial recanalization of the right portal vein branches, without complications. New DOACs do not need daily subcutaneous injections nor routinely blood coagulation control tests, making its use attractive, eventually increasing patient's compliance. If proved to be safe and effective in the future studies, its use may be extended to PVT treatment. This case shows that rivaroxaban was safe, not only prevented the extension of thrombosis in the portal tract, but also resolved PVT, at least partially.
\end{abstract}

Keywords: Portal vein thrombosis; Anticoagulation; Direct-acting oral anticoagulants

\section{Introduction}

Portal vein thrombosis (PVT), an event that is associated with

\section{Manuscript accepted for publication March 01, 2017}

aServico de Cuidados Intensivos - Unidade Intermedia Medica, Centro Hospitalar do Porto, Hospital Sto Antonio, Porto, Portugal

bEpiUnit - Instituto de Saude Publica da Universidade do Porto, Porto, Portugal

'Instituto de Ciencias Biomedicas de Abel Salazar - UP, Porto, Portugal

dServico de Hematologia - Centro Hospitalar do Porto, Hospital Sto Antonio, Porto, Portugal

eServico de Radiologia, Centro Hospitalar do Porto, Hospital Sto Antonio, Porto, Portugal

${ }^{f} H$ Hepatology, Inselspital, Berne, Switzerland

${ }^{g}$ Corresponding Author: Filipe Nery, Servico de Cuidados Intensivos - Unidade Intermedia Medica, Centro Hospitalar do Porto, Hospital Sto Antonio, Largo Prof. Abel Salazar, S/N 4000-001 Porto, Portugal.

Email: filipegaionery@gmail.com

doi: https://doi.org/10.14740/gr806w a large spectrum of clinical presentations and severity, may be related to cirrhosis, malignancy, local factors or idiopathic [1]. A diagnostic workup to identify an underlying pro-thrombotic condition must be undertaken. In acute portal vein thrombosis (APVT) unrelated to cirrhosis, early anticoagulant treatment is indicated in order to achieve recanalization and, therefore, avoid future complications [1]. With conventional anticoagulant treatments (low molecular weight heparin (LMWH) or vitamin $\mathrm{K}$ antagonists), recanalization occurs in up to one-third of patients, being more difficult to achieve when ascites or occluded splenic vein are present [2]. In contrast, if anticoagulation is not started, recanalization is exceptional $[1,3]$. The use of DOACs is being generalized [4], but they are not routinely used in PVT and are not yet mentioned in the latest guidelines as an alternative treatment [1]. Recent data, however, indicate that this new class of anticoagulants is increasingly used also in patients with splanchnic vein thrombosis $[5,6]$.

\section{Case Report}

A 28-year-old healthy female, active smoker of 5 pack-year and under oral contraceptive (ethinylestradiol and gestodene) presented to the emergency department with severe epigastric pain of 5-day duration. No nausea, vomiting, diarrhea or bloody stools were reported; she was afebrile, hemodynamically stable and physical examination was normal except for pain on the upper quadrants without signs of peritoneal irritation or ascites. Renal function and liver profile were normal; $\mathrm{CBC}$ revealed only discrete anemia (hemoglobin of $11.8 \mathrm{~g} / \mathrm{dL}$ ), with normal platelet count; D-dimers were elevated (948 ng/ $\mathrm{mL}$ ), INR and partial thromboplastin time were within the normal range; C-reactive protein was elevated $(64.18 \mathrm{mg} / \mathrm{L})$ with no infection focus found. Abdominal ultrasound with Doppler revealed thrombosis of more than $50 \%$ of the lumen of both right and left portal branches with no extension to the portal vein trunk, superior mesenteric or splenic veins. A computed tomography (CT) scan confirmed those findings with normal liver morphology and no ascites. Upper endoscopy revealed no portal hypertension-related complications. The patient was admitted at the intermediate care unit and unfractionated heparin for $24 \mathrm{~h}$ was started with a subsequent relay to LMWH (enoxaparin) in the dose of $1 \mathrm{mg} / \mathrm{kg}$ twice daily. Two days after she was discharged, and after discussion and agreement with the 


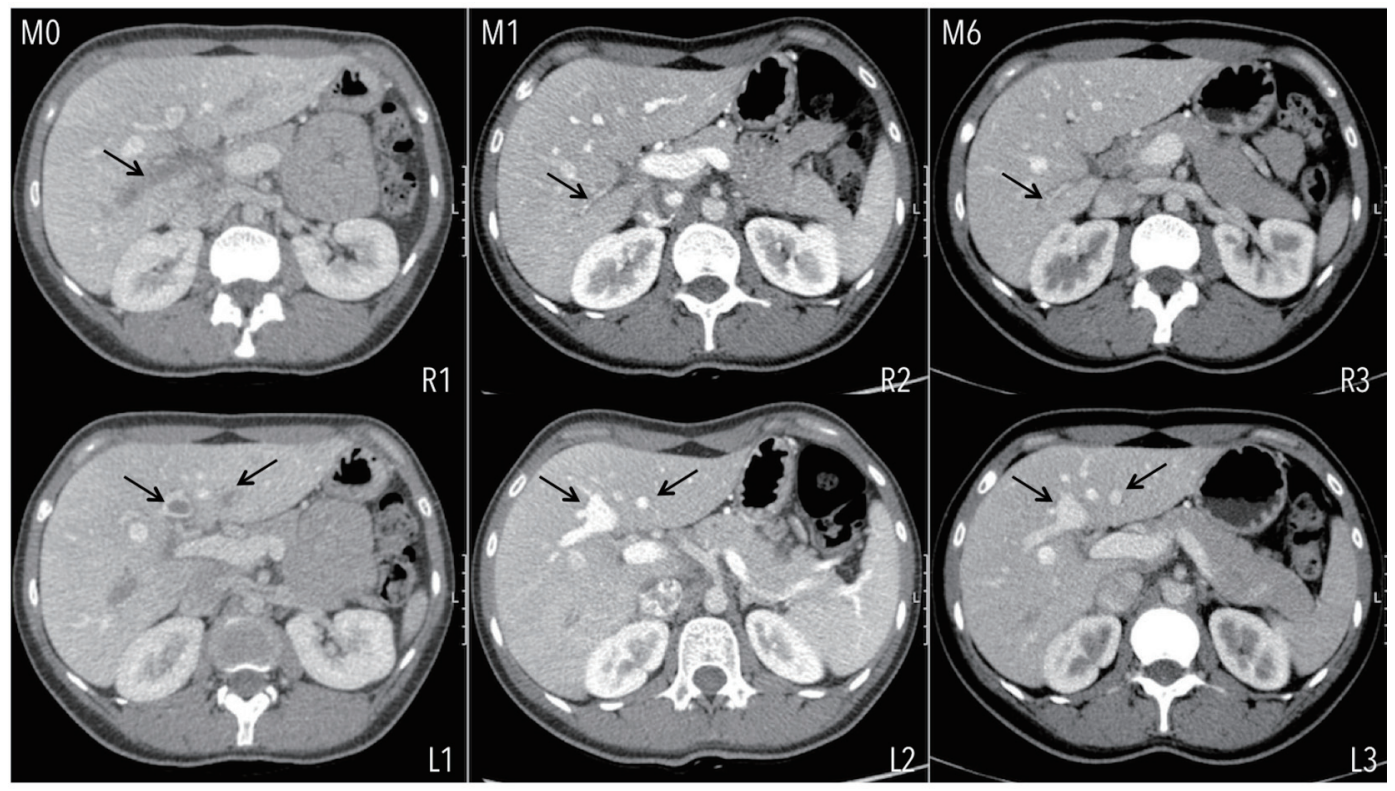

Figure 1. Extension of PVT at diagnosis (M0), involving the totality of the right (R1) and segments of the left portal vein branches (L1). Evolution of PVT under rivaroxaban at the end of the first (M1) and sixth (M6) months of treatment. Total recanalization is seen at the left portal vein branch and partial at the right portal vein branch.

patient, she received rivaroxaban treatment $15 \mathrm{mg}$ twice daily. By that time she was nearly asymptomatic and instructed to stop smoking and to substitute previous oral contraceptive for a progestative one. Treatment was continued for 3 weeks and then $20 \mathrm{mg}$ daily. The 1-month CT scan control showed total recanalization of the left branch and persistence of right branch thrombosis but with partial recanalization. No extension of the thrombus was documented (Fig. 1). Those findings were similar in the 6-month CT scan. Pro-thrombotic workup diagnosis revealed negativity for JAK2 and calreticulin mutations, normal homocysteine serum levels, absence of lupus anticoagulant and negativity for paroxysmal nocturnal hemoglobinuria. Anti-cardiolipin, anti- $\beta 2$ glycoprotein I, anti-prothrombin and anti-phosphatidylserine antibodies were all negative. Factor VLeiden and prothrombin G20210A gene mutations were both found to be positive in heterozygosity. The patient remains under rivaroxaban $20 \mathrm{mg}$ daily, asymptomatic with normal renal function and liver profile with no documented complications of the anticoagulant treatment.

\section{Discussion}

To our knowledge, this is the first case ever reported concerning treatment with DOACs for a patient with APVT unrelated to cirrhosis. Three other reports using rivaroxaban to treat APVT were published, but all of them in patients with underlying chronic liver diseases [7-9]. In all, total recanalization of the affected segments was achieved after a 1-, 6- and 3-month treatment period, respectively. Rivaroxaban doses varied between 10 and $20 \mathrm{mg}$ daily. More recently, another successful case of rivaroxaban use for a recurrent PVT in a patient with a Child-Pugh class A cirrhosis, with total recanalization was published [10]. In all described cases, none complication related to rivaroxaban use was documented. In cirrhosis, the use of either direct thrombin inhibitors, as dabigatran, or direct factor Xa inhibitors, as rivaroxaban, apixaban or endoxaban, may be, at least in theory, even more effective than LMWH or vitamin $\mathrm{K}$ antagonists. This because it has been postulated that lower portal flow velocities, which may raise the risk of PVT development, may lead to a lower wash out of active thrombin generated in portal circulation and thus contribute to a "local" higher prothrombotic environment [11]. Other major concern about DOACs use is also its safety, since they all are metabolized in the liver [4].

According to current EASL recommendations, treatment in the setting of APVT unrelated to cirrhosis should be with unfractionated heparin or LMWH in therapeutic doses with a subsequent substitution for a vitamin $\mathrm{K}$ antagonist targeting an INR between 2 and 3 [1]. In this particular case, and under rivaroxaban treatment, a rapid and total recanalization was achieved in left portal branch but not completely in the right, probably linked to a more "occlusive" thrombosis in the latter. Also, in our patient, rivaroxaban proved to be safe, with no bleeding complications reported to date. The dose of rivaroxaban chosen was the one indicated for deep vein thrombosis and pulmonary embolism, which was higher than in almost all of the other case reports, but this patient did not have concomitant liver disease nor acute decompensation/failure. Also, rivaroxaban was started 2 days after LMWH was started, as one of the options suggested by recommended guidelines for acute pulmonary embolism [12]. This may reflect a more cautions strategy in the acute setting of PVT, allowing the management of potential complications that may arise in the first hours of hospitalization with the medications that physicians are more used to. 
Two series have been published so far reporting the use of DOACs in patients with splanchnic vein thrombosis $[5,6]$. The results of these two papers strongly suggest that this new class of anticoagulants is safe, since the incidence of major bleedings and other drug-induced side effects was similar as in patients treated with conventional anticoagulation. Since DOACs are increasingly used off label in this indication, more data about safety and efficacy are eagerly needed.

This case report suggests that the treatment of APVT in a patient without underlying liver disease can 1) prevent the extension of the thrombus in the portal tract; 2) resolve PVT, at least partially, and most likely when a non-occlusive thrombosis occurs and; 3) be safe. In this setting, randomized controlled studies are needed in order to assess efficacy and safety of DOACs compared to LMWH or vitamin K antagonists before its generalization.

\section{Conflicts of Interest}

None.

\section{Funding}

None.

\section{Consent}

Informed consent was obtained from patient.

\section{Guarantor}

$\mathrm{FN}$ is the guarantor of this article.

\section{References}

1. EASL Clinical Practice Guidelines: Vascular diseases of the liver. J Hepatol. 2016;64(1):179-202.

2. Plessier A, Darwish-Murad S, Hernandez-Guerra M, Con- signy Y, Fabris F, Trebicka J, Heller J, et al. Acute portal vein thrombosis unrelated to cirrhosis: a prospective multicenter follow-up study. Hepatology. 2010;51(1):210218.

3. Condat B, Pessione F, Helene Denninger M, Hillaire S, Valla D. Recent portal or mesenteric venous thrombosis: increased recognition and frequent recanalization on anticoagulant therapy. Hepatology. 2000;32(3):466-470.

4. Roca B, Roca M. The new oral anticoagulants: Reasonable alternatives to warfarin. Cleve Clin J Med. 2015;82(12):847-854.

5. Intagliata NM, Henry ZH, Maitland $\mathrm{H}$, Shah NL, Argo CK, Northup PG, Caldwell SH. Direct Oral Anticoagulants in Cirrhosis Patients Pose Similar Risks of Bleeding When Compared to Traditional Anticoagulation. Dig Dis Sci. 2016;61(6):1721-1727.

6. De Gottardi A, Trebicka J, Klinger C, Plessier A, Seijo S, Terziroli B, Magenta L, et al. Antithrombotic treatment with direct-acting oral anticoagulants in patients with splanchnic vein thrombosis and cirrhosis. Liver Int. 2016.

7. Pannach S, Babatz J, Beyer-Westendorf J. Successful treatment of acute portal vein thrombosis with rivaroxaban. Thromb Haemost. 2013;110(4):626-627.

8. Martinez M, Tandra A, Vuppalanchi R. Treatment of acute portal vein thrombosis by nontraditional anticoagulation. Hepatology. 2014;60(1):425-426.

9. Lenz K, Dieplinger B, Buder R, Piringer P, Rauch M, Voglmayr M. Successful treatment of partial portal vein thrombosis (PVT) with low dose rivaroxaban. Z Gastroenterol. 2014;52(10):1175-1177.

10. Yang H, Kim SR, Song MJ. Recurrent acute portal vein thrombosis in liver cirrhosis treated by rivaroxaban. Clin Mol Hepatol. 2016;22(4):499-502.

11. Zocco MA, Di Stasio E, De Cristofaro R, Novi M, Ainora ME, Ponziani F, Riccardi L, et al. Thrombotic risk factors in patients with liver cirrhosis: correlation with MELD scoring system and portal vein thrombosis development. J Hepatol. 2009;51(4):682-689.

12. Konstantinides SV, Torbicki A, Agnelli G, Danchin N, Fitzmaurice D, Galie N, Gibbs JS, et al. 2014 ESC guidelines on the diagnosis and management of acute pulmonary embolism. Eur Heart J. 2014;35(43):3033-3069, 3069a-3069k. 